\title{
VASCULAR PATTERNS IN THE ENDOMETRIUM AND THE PLACENTA
}

\author{
ELIZABETH M. RAMSEY, M.D. \\ INTRODUCTION
}

At a conference dealing with research in small blood vessels a report of work upon the circulation of the placenta may seem to be insinuating itself under false pretenses, for the placenta can hardly be described as a "small" vascular channel, least of all the placenta of primates.

However, if the physiology of the placenta is considered rather than its gross morphology, it is at once apparent that what the placenta does is identical with what microscopic blood vessels do, such as may be studied in the rat's mesentery or the hamster's cheek pouch or the wing membranes of the bat, for all are essentially terminal areas of metabolic exchange. To describe the vast intervillous space of the monkey or human placenta as a capillary bed may cause an initial shock of surprise but, after a moment's mental readjustment, the concept proves a valid and satisfying one and to the present Conference, concerned as it specifically is with "vascular patterns as related to function," the primate placenta can speak directly. It speaks not only of the interrelationship of vascular structure with function, but of the ability of blood vessels to adapt to alterations in the function of the tissues they supply, as illustrated by the remarkable changes exhibited by endometrial blood vessels throughout the various phases of the menstrual cycle and under the special circumstances of pregnancy. In addition, although the techniques of investigation have been conventional, the work to be reported introduces an unconventional object for vascular research which may open up some new horizons of thought or suggest fresh avenues for exploration.

The relationships in the hemochorial placenta of primates are shown in figure 1. It is seen that maternal arteries traverse the uterine wall and terminate in the intervillous space of the placenta just as systemic arteries terminate in systemic capillaries, and that maternal veins drain the space as systemic veins drain capillaries. This analogy is supported by the histological characteristics of the walls of the components of the placental circulation, as will be brought out later on. Pursuing the analogy into the physiological field, it is seen that metabolic exchange occurs across the wall of the intervillous space just as it does across the wall of any capillary. The trophoblast covering the chorionic villi takes the place of capillary endothelium and the mesoderm of the villi forms the interstitial tissue through which metabolites pass to and from the fetal capillaries.

Having thus established the legitimacy of the placenta's claim to kinship with the "small vessels" of the body, attention may be directed first to its maternal and then to its fetal vascular components and finally to their interrelations.

\section{MATERNAL PLACENTAL CIRCULATION}

The menstrual cycle

One cannot discuss the maternal placental vasculature in primates without considering first the behavior of endometrial blood ressels in the non-pregnant 


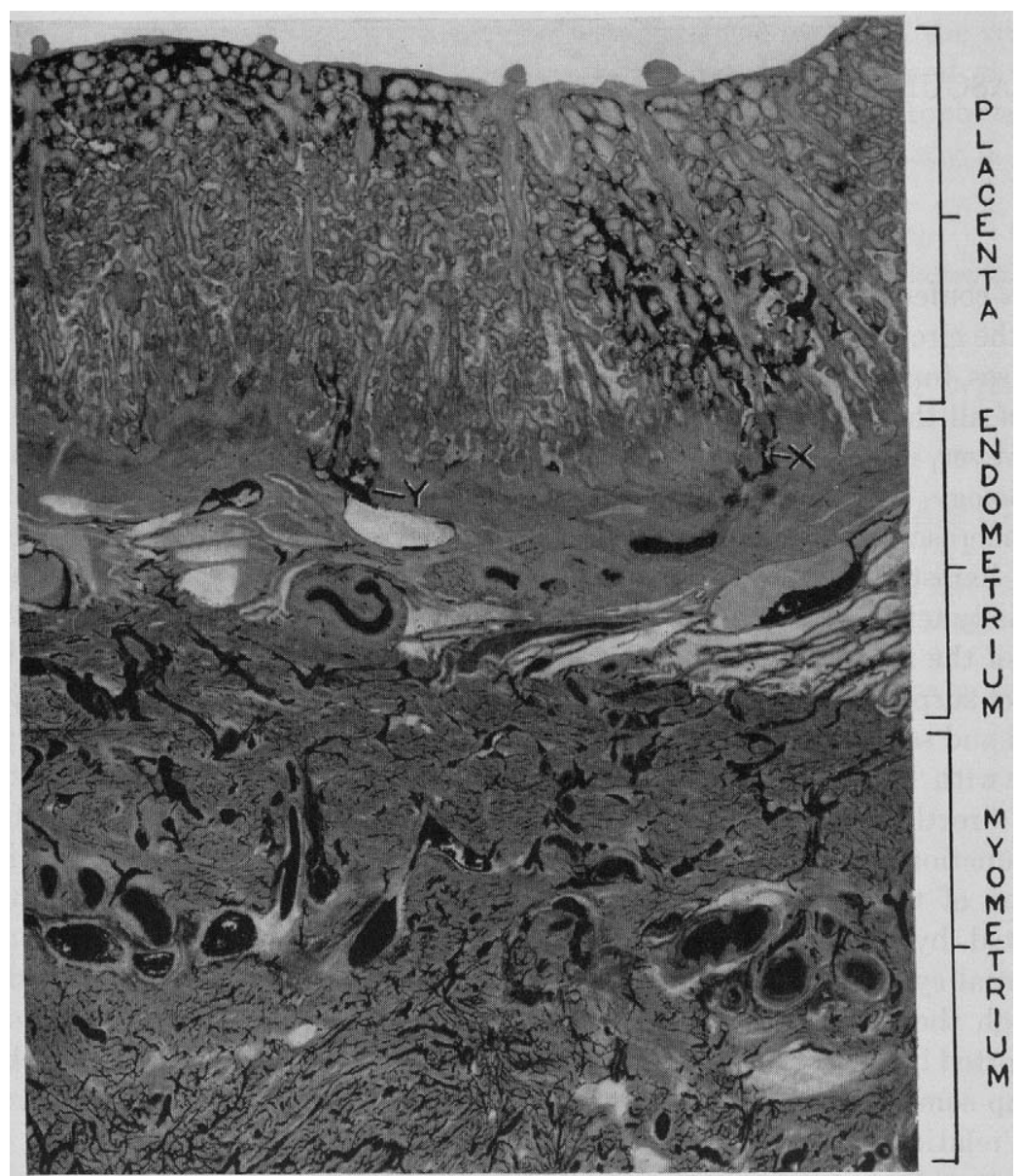

FIG. 1. An over-all view of part of the uterine wall and attached placenta in a monkey 38 days pregnant. Maternal arteries and veins are seen traversing the myometrium and endometrium. An arterial entry to the intervillous space is seen at $x$ and a venous exit at $\mathrm{y}$. India ink injection. C $658 . \times 10$.

cycle. These vessels conform in more familiar and conventional fashion to the microcirculatory category and so it is good for two reasons to start with them.

Arterial blood reaches the primate uterus from the aorta via the uterine arteries, and some branches of the ovarian arteries, which course through the broad ligaments bilaterally and upon arrival at the uterine wall plunge at once into the middle layer of the myometrium where they break up into branches and form a circumferential wreath of arcuate arteries. The relationships are shown in figure 2. Radial arteries coming off the arcuates at a sharp angle penetrate the junction between myometrium and endometrium and divide into two distinctly different sorts of endometrial arteries; the basals, which are relatively insensitive to hormonal stimulation and show no striking changes during the menstrual cycle, and the spiral arteries, which coil upward toward the endo- 


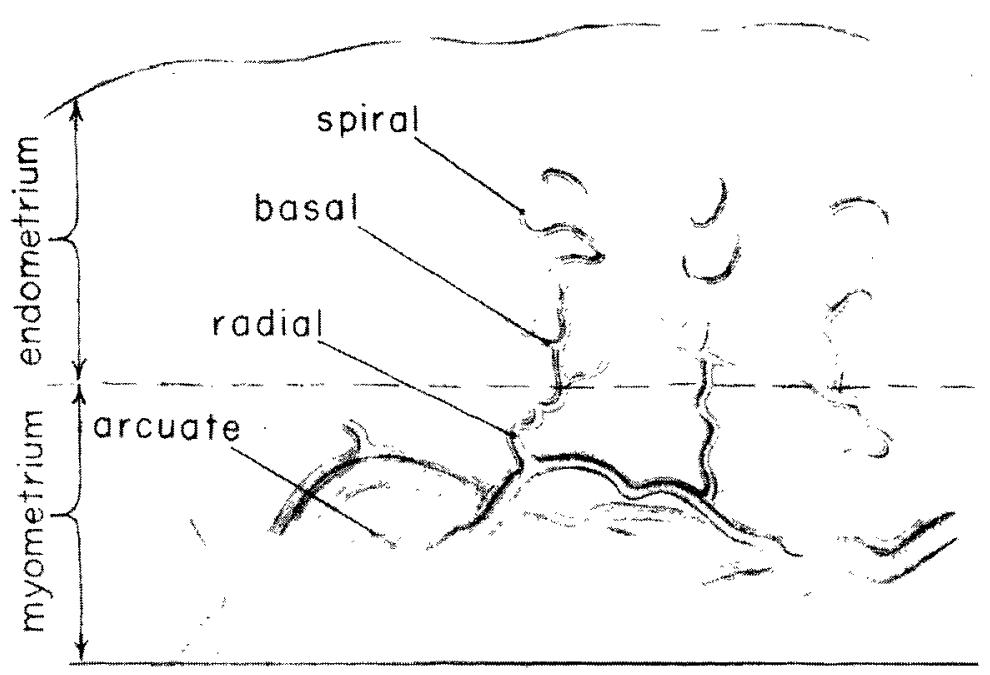

FIG. 2. A schematic representation of uterine arteries after Okkels and Engle (13).

metrial surface, responding to the stimulation of the ovarian hormones as the cycle progresses. The intravital injections of dyes in monkeys by Daron $(1,2)$, with subsequent study of serial sections, the intraocular transplants of endometrium performed by Markee (3) in monkeys, and the extensive studies of Bartelmez (4-6) in both the human and the monkey are the classic studies upon which is based the following theory of the mechanism of menstruation. It will be seen by comparing figures 3 and 4 that as the cycle progresses the spiral arterioles increase in length, grow ever closer to the surface epithelium, and become more and more coiled. If pregnancy does not supervene, hormonal support is withdrawn and the endometrium regresses as the result of the removal of intra- and extracellular fluid. This occasions a decrease in endometrial thickness with consequent increased coiling of the arterioles. The next phase is characterized by intense ischemia leading to necrosis and to the slough and hemorrhage of menstruation. Studies which Dr. Bartelmez is currently preparing for publication (7), but kindly allows me to mention at this time, make it clear that the causative factor underlying the ischemia is localized constriction of the spiral arterioles by intrinsic contraction of the smooth muscle in their walls and not the increased coiling of the arterioles, which has previously been inroked as the causative factor.

In addition to the arterial features already mentioned, the venous pattern of the endometrium must be considered. This is characterized essentially by a superficial and a deep chain of dilated lakes which are connected with each other and with the venous plexus in the myometrium by numerous radial venules. A very rich capillary network lies in the subepithelial layer of the endometrium. With respect to the claims that arterio-venous anastomoses are present in the 


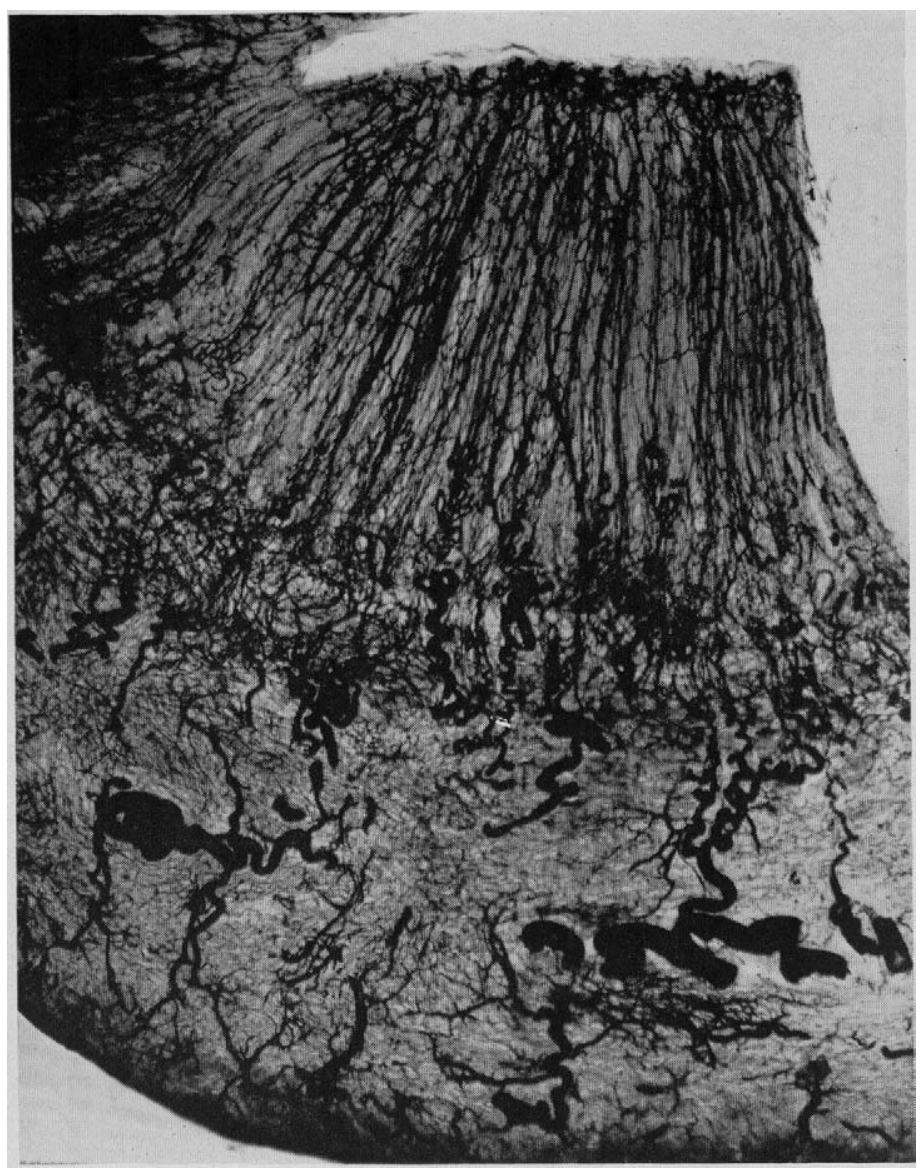

Fig. 3. A cross section of the uterine wall of a monkey on day 12 of the menstrual cycle. A mature follicle was demonstrated in the ovary. A spiral arteriole displays minimal coiling in the lower third of the endometrium. Its straight distal end reaches half way to the surface epithelium. India ink and starch injection. B 338. $\times 15$. Kindness of Dr. G. W. Bartelmez.

endometrium, it can now be stated that if standard anatomical criteria are rigorously and objectively applied to the data, very serious doubts are raised as to the existence of such structures. This important matter is also under investigation by Dr. Bartelmez (7).

\section{Pregnancy}

If pregnancy occurs, the fertilized egg of the monkey enters the endometrium about the ninth post-ovulatory day (that of man probably a day or two earlier), and its first nourishment is obtained from local pabulum (8). Very soon, however, the trophoblast invades maternal blood channels, initiating changes which have been studied in primates at the Carnegie Embryological Laboratory over the past 10 years according to the following experimental techniques (9-11). 


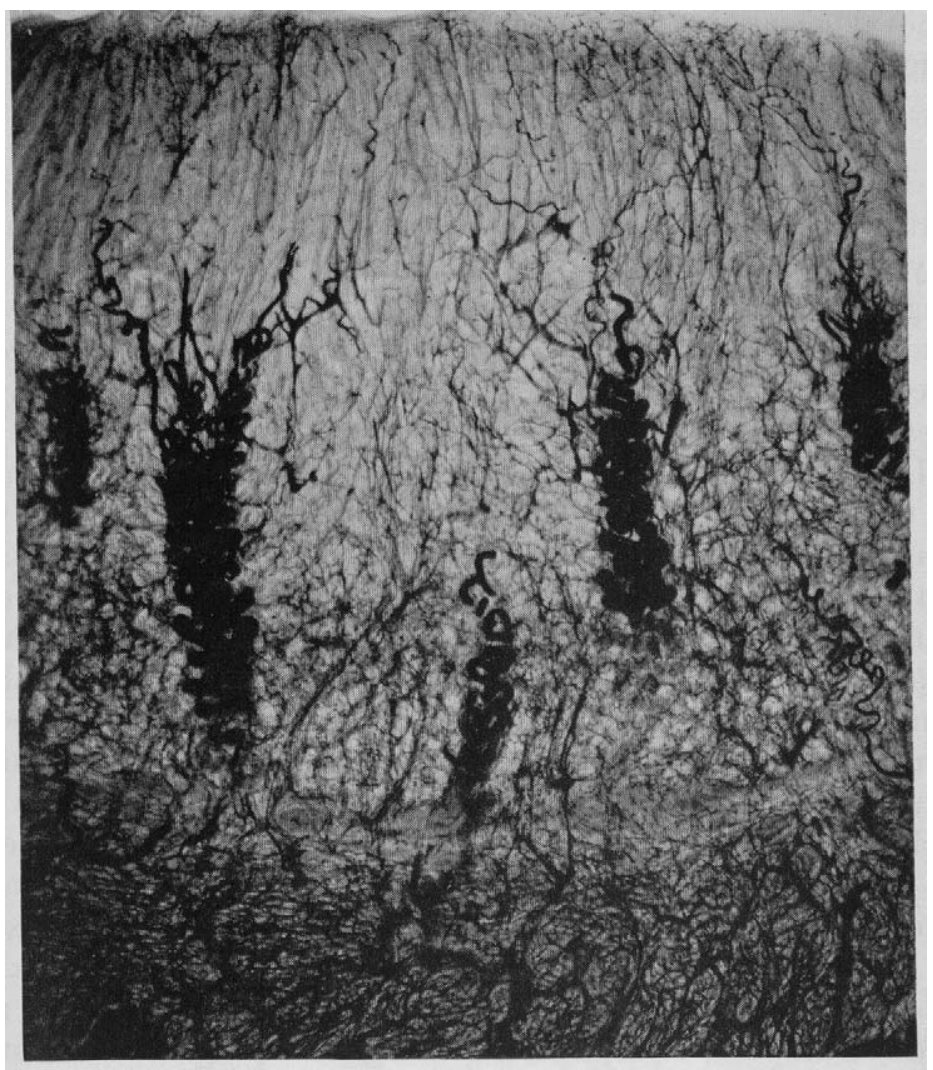

Fig. 4. A cross section of the uterine wall of a monkey on day 17 of the menstrual eycle. A 6-day-old corpus luteum was demonstrated in the ovary. The abundant coils of three arterioles are seen in the middle third of the endometrium. The straight distal ends reach $3 / 4$ of the way to the surface epithelium. India ink and starch injection. B 309. $\times 15$. Kindness of Dr. G. W. Bartelmez.

\section{Materials and methods}

In a group of 23 rhesus monkeys (Macaca mulatta) which had been palpated for ovulation, bred, and maintained for desired periods representative of all stages of gestation, intra-aortic injections of India ink were carried out in the anesthetized animal upon the desired day of pregnancy. Subsequently the animal was killed and the entire genital tract removed and fixed en bloc. Serial sections were prepared of numerous areas of the uterine wall and attached placenta and three-dimensional models of the vasculature were constructed as indicated.

The human material, comprising 20 cases to date, was all obtained at hysterectomy for systemic disease except for two specimens removed at autopsy. Representative stages of pregnancy are already included in the series and specimens 
of duplicate ages are now being collected for corroboration. The uteri were perfused and injected with India ink through the uterine arteries within minutes after removal. Fixation, serial sectioning, and modelling have been carried out in the same manner as with the monkey material.

\section{Results}

As the trophoblast of the implanting blastocyst invades the surrounding endometrium it erodes first the maternal capillaries, then the veins, and finally, about day 19, the tips of the spiral arterioles. These vessels pour their blood into the young intervillous space of the placenta. Thereafter, continued growth of the trophoblast opens up progressively more spiral arterioles which accommodate themselves to the reduction in endometrial thickness, occasioned by pressure and encroachment of the overlying conceptus, by increased coiling and back-andforth and lateral looping. This factor is augmented by increase in the absolute length of the arteries during the period of growth. These features are illustrated in the diagram in figure 5, which is based on three-dimensional reconstructions of placental arteries in the monkey. The phenomena are very closely duplicated in the human. The first three drawings in this figure, based on specimens in the early stage of pregnancy, show the progressive decrease in endometrial thickness and the increased coiling and looping of the arteries. Then, at about the ninetieth day of pregnancy in the monkey and in the human around the twentieth week, there occurs the great transformation known as "conversion," first described by Reynolds (12). This occurs when accommodation of the products of conception ceases to be effected primarily by uterine growth and commences to be based upon uterine stretching. During the period of stretching, the coils of spiral arterioles are paid out much as reserves of rope coiled up on the deck of a boat are paid out as needed. The beginning and final stages of this process are shown in the last two drawings in the figure. The role of the coiling of the endometrial arteries is thus made clear: it exists not to initiate the destructive changes at the end of the menstrual cycle, but to fulfill the continuing demands of uterine enlargement after the possibility of further growth has ceased. This is a dramatic example of that "adaptation of pattern to changing function" referred to earlier as a pre-eminent characteristic of placental vasculature. How the adaptation is effected will be noted in the discussion of the structure of the walls of the placental vessels.

The responses to pregnancy exhibited by endometrial veins are shown in figure 6 , which presents models of the veins occurring in the same tissue blocks as the arteries in the previous diagram. The veins, it is seen, adapt to both the growth and the stretching phases of uterine enlargement chiefly by passive alterations in caliber. In addition, the absolute number of connections between the intervillous space and maternal veins is markedly reduced as pregnancy advances, though some persist in all regions of the placenta, centrally as well as marginally. Excursion into the interesting detour provided by this fact will be avoided, note merely being taken at this point that a hemodynamic mechanism of placental circulation is suggested by the present study, superseding earlier 

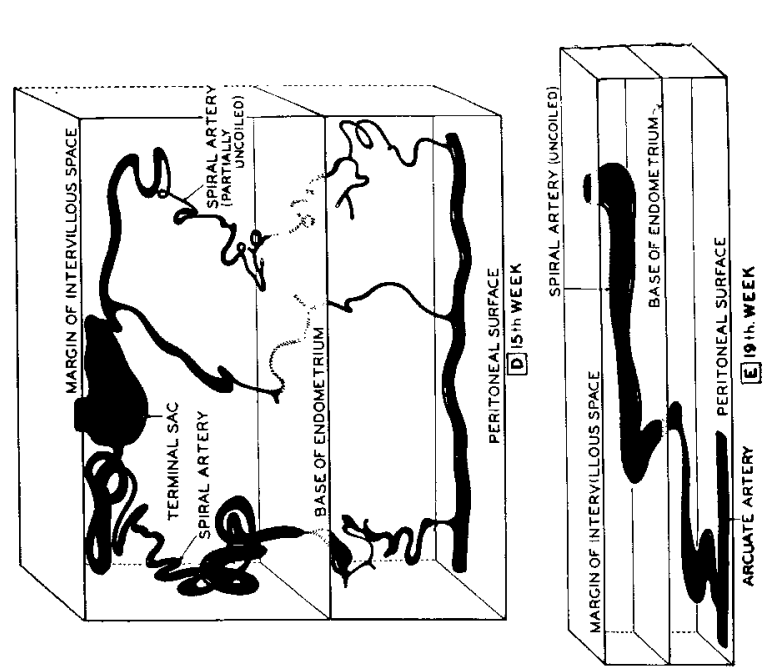

$\stackrel{8}{ \pm}$

t艹

节

.

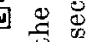

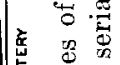

它

岁范

买

留

융

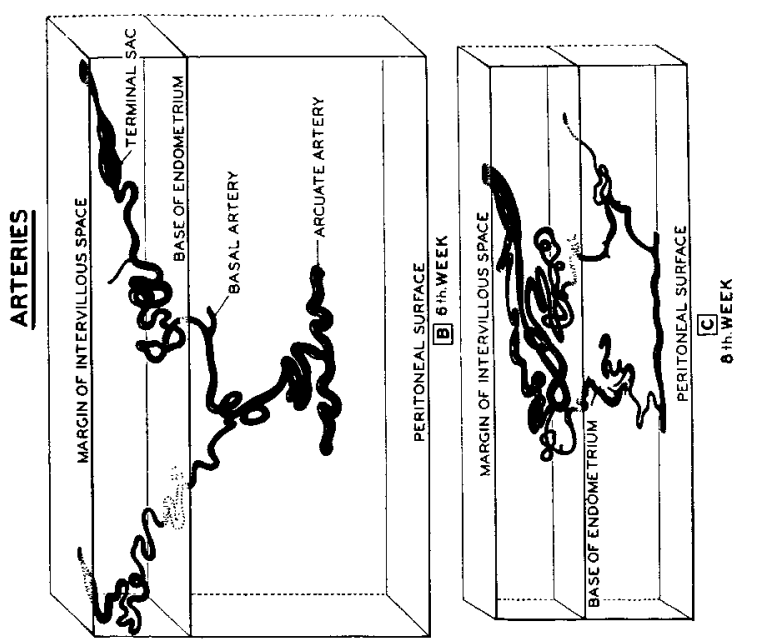

รี

궁

敂莺

苛

$0+$

훙

용

范

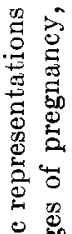

害营

范

여ำ

蛋

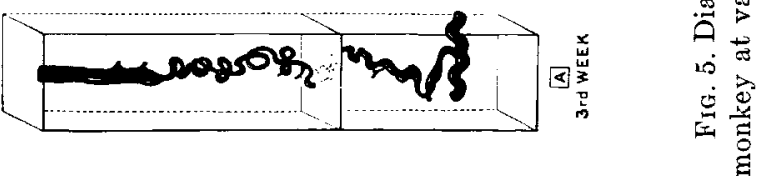




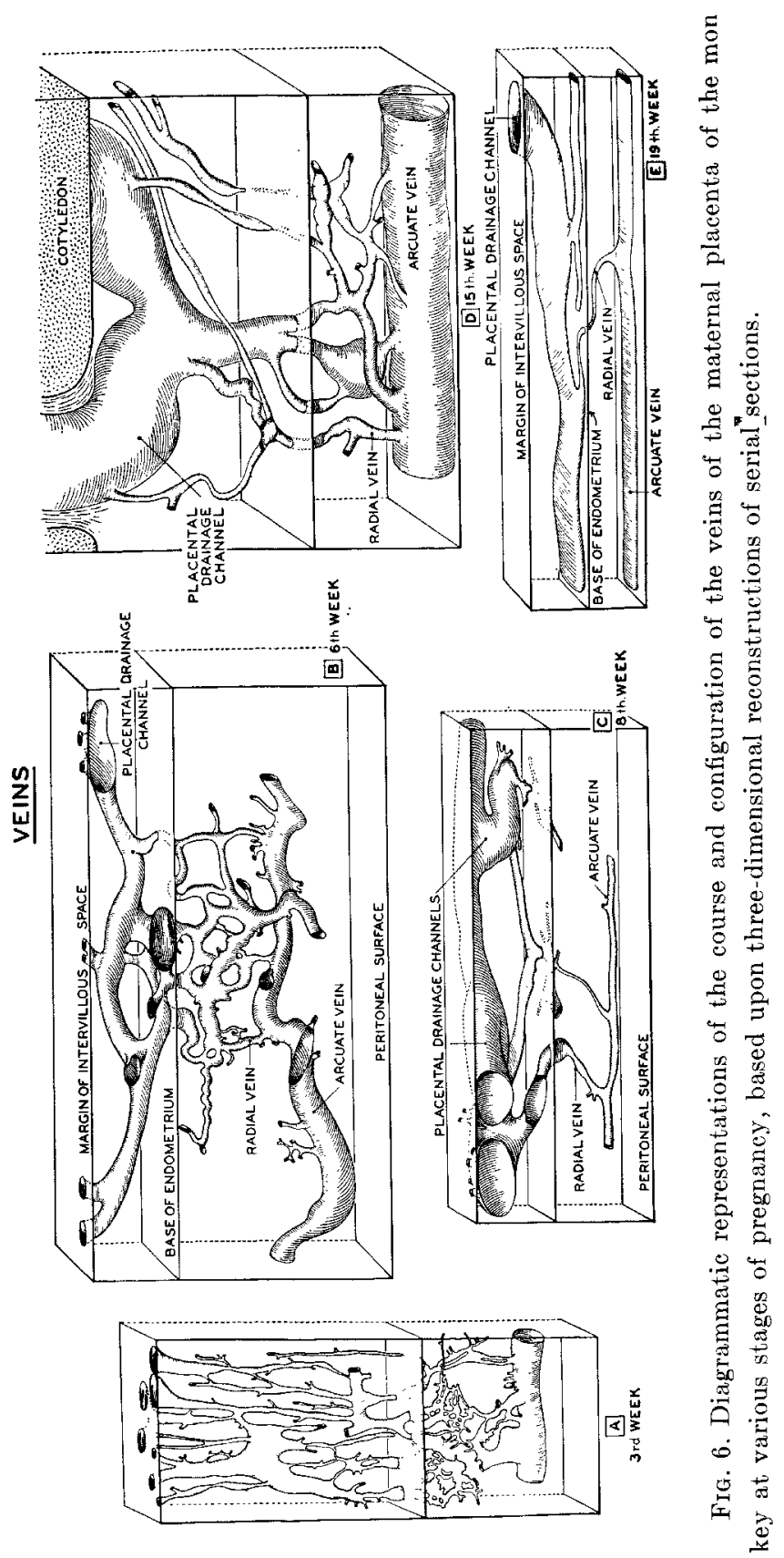


theories which postulated mechanical arrangements unconfirmed by more recent investigations.

The structure of the walls of the vessels whose course has been traced must be considered before turning to examination of the fetal side of the placenta. The veins may be summarily dismissed with the statement that they are similar to their relatives elsewhere in the body, except that they lack valves, and their histological constitution remains unchanged throughout the cycle and pregnancy. The obliteration of some of the abundant early connections with the intervillous space, which was mentioned above, appears to be effected altogether by external compression.

To the placidity of the veins is opposed the dynamic activity of the endometrial arteries. It cannot be said that they are ever in a true "resting state," but in their basic form their walls are similar to those of the radial arteries with which they are contimuous, containing abundant coarse elastic tissue distributed as membranes between the intima and media, as scattered fibrils in the latter and again as an irregular membrane in the adventitia (13). A fibrillar elastic network persists after the caliber has been reduced to the point where the designation "arterioles" is appropriate and may even extend to the subepithelial region. Mitoses and growth phenomena are prominent throughout the menstrual cycle, lasting until shortly before onset of bleeding. The earlier theory that the distal portions of the vessels down to the level of the basalis are destroyed and sloughed off at the time of menstruation is no longer considered valid (7). The subepithelial capillary bed is probably so destroyed and the tips of the arterioles may be frayed for a variable but short distance, but basically their integrity is retained.

If pregnancy occurs, the hormonal support of the endometrium is, of course, maintained and in place of the regressive changes heralding menstruation, there is a continuation of growth of the spiral arterioles toward the epithelial surface. When the tips of the arterioles are tapped by the invading trophoblast, a wave of endothelial proliferation is initiated, commeneing in the region of transition into the intervillous space and gradually progressing proximally almost to the myo-endometrial junction. Only vessels which open into the placenta, the socalled "placental arteries" proper, are so affected (figs. 7 and 8). Hand in hand with the endothelial change goes an alteration in the media. This first attracts notice in the terminal portion of the artery which, as seen in figure 8 , gradually dilates to form a small sac in which the media and adventitia seem to be replaced by the proliferated lining cells, an appearance confirmed by specific stains which demonstrate the absence of elastic fibers. In the deeper layers of the endometrium, normal media and adventitia persist, even in the presence of endothelial proliferation. About the end of the first third of pregnancy, the endothelial proliferation wanes and the exuberant disorder of the early stages settles down to a compact and regular palisading and the cells show increasing maturity. At the same time the muscle cells of the media, in the portions of the artery proximal to the terminal sac, undergo an epithelioid transformation which is observed at ever greater depths until eventually the myometrial radial arteries are involved and, just before term, the myometrial arcuate stems (fig. 9). The final histological 


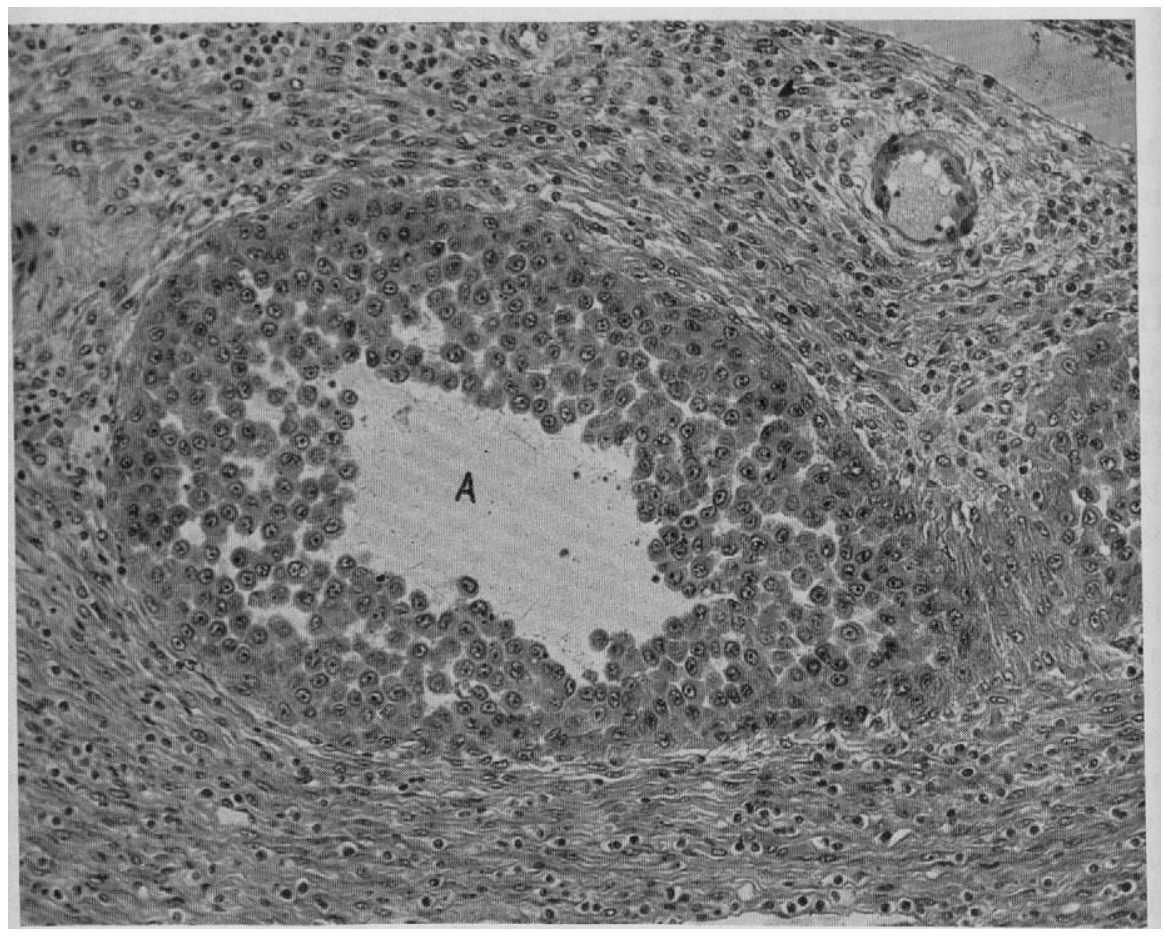

FIG. 7. Extensive endothelial proliferation is seen at A in a spiral artery just before its entrance into the intervillous space. Monkey $\mathrm{C} 477$, twenty-ninth day of pregnancy. $\times 150$.

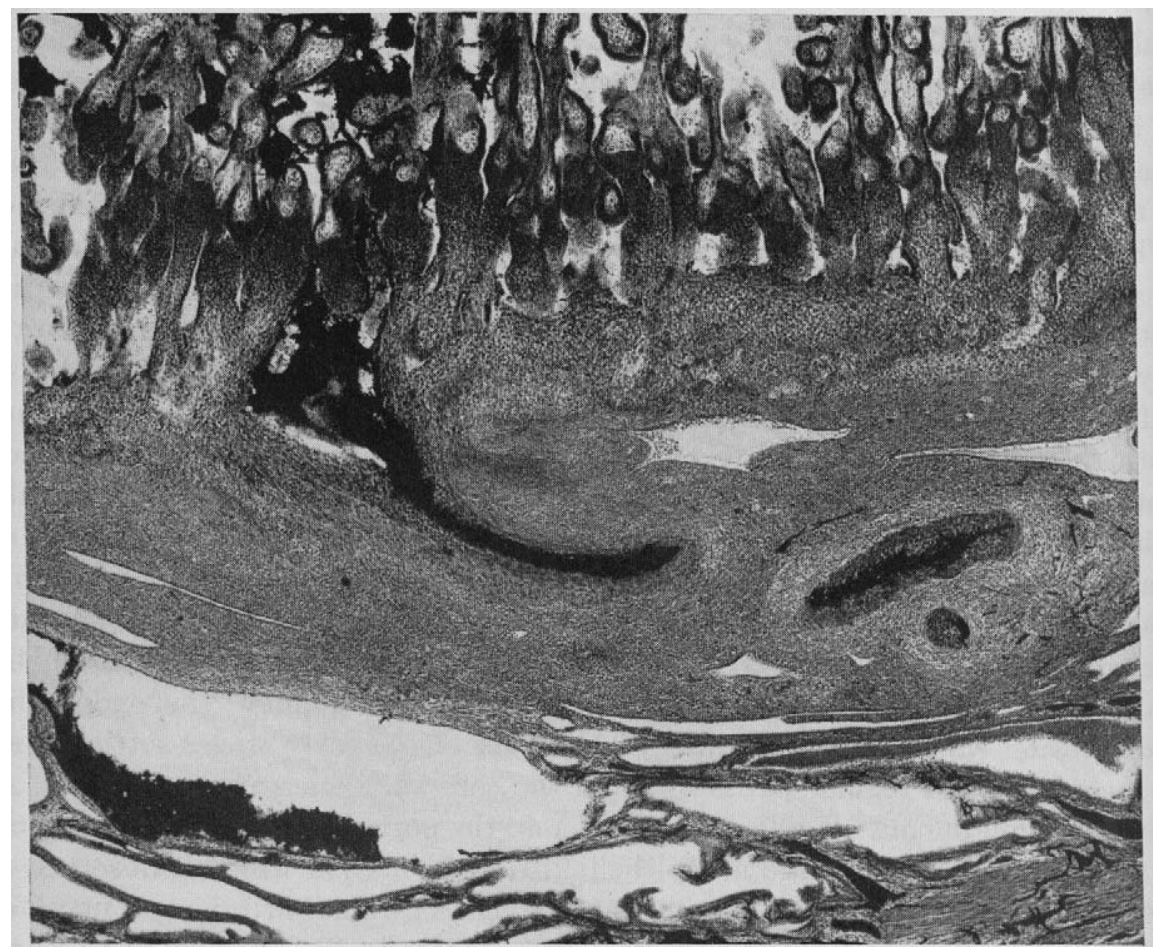

FIG. 8. A spiral artery is seen at its point of entry to the intervillous space (left) and two cross sections of a more proximal coil of the same vessel are seen at the right. Pronounced endothelial proliferation is apparent and there is beginning epithelioid transformation of the cells of the media. Monkey $\mathrm{C} 658$, thirty-eighth day of pregnancy. $\times 30$. 


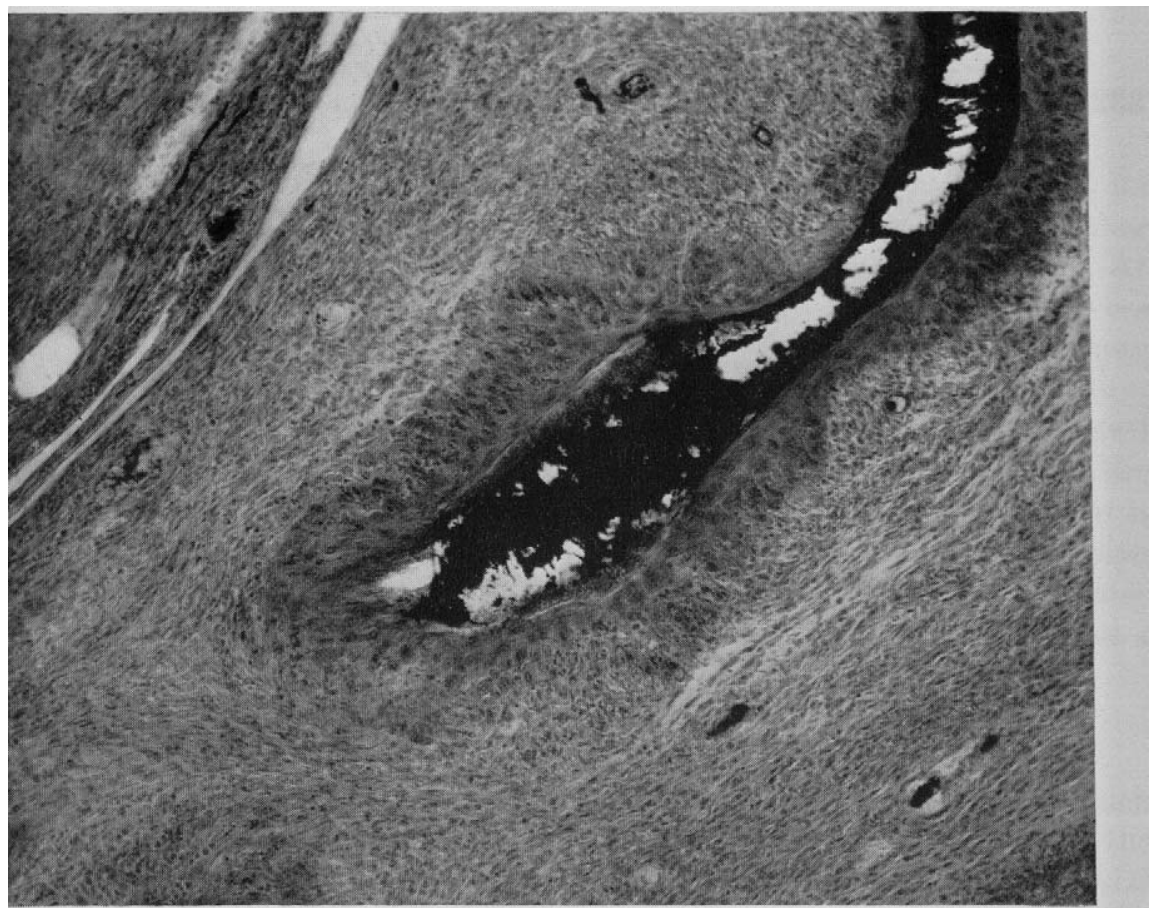

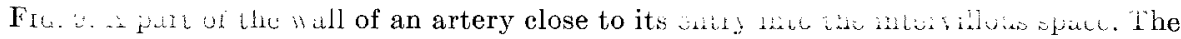
terminal dilatation into a small sac is seen and the epithelioid transformation of the cells of the media. Endothelial proliferation has already waned. Monkey C 629, fifty-third day of pregnancy. $\times 100$.

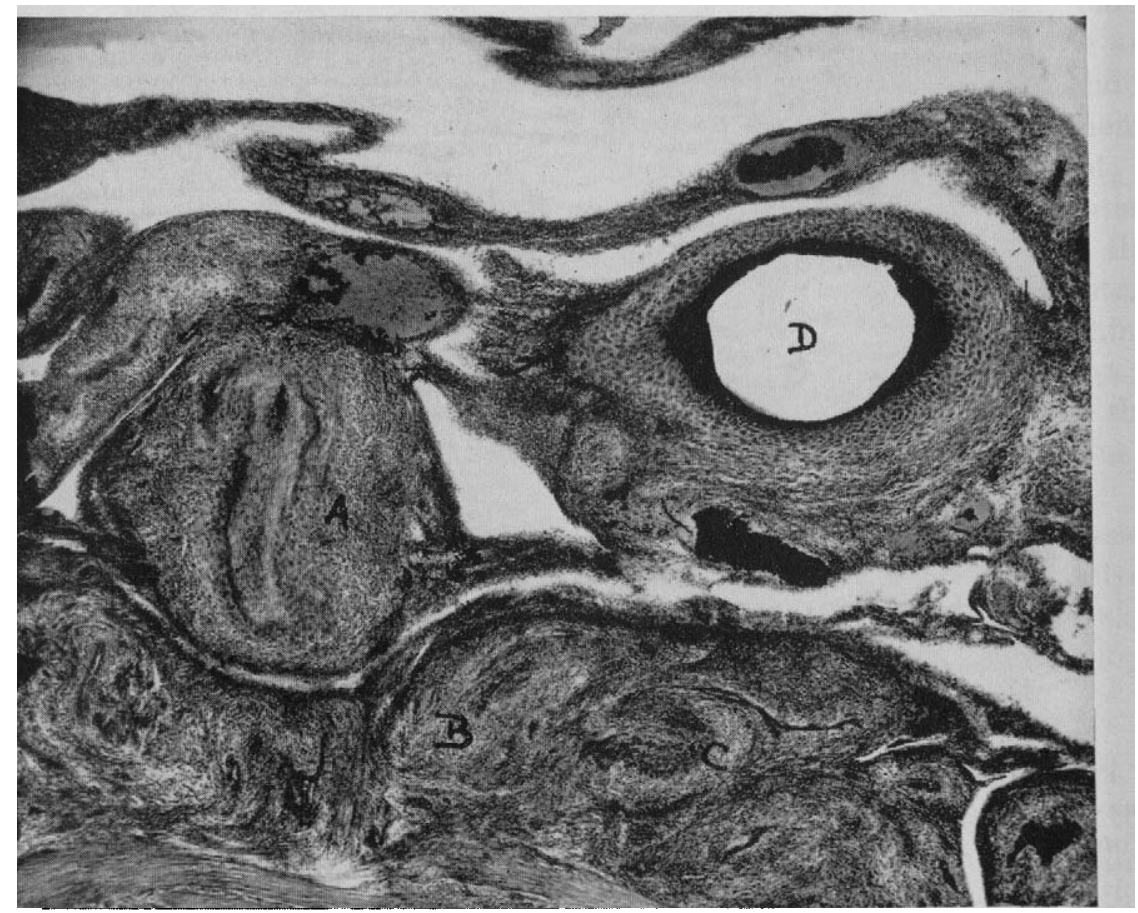

FIG. 10. Intimal fibrosis almost completely occludes the lumen of an artery in the midendometrium (A); constriction of an arterial lumen without intimal fibrosis is seen at $\mathbf{B}$ and $\mathrm{C}$; epithelioid change in the media of an artery appears at $\mathrm{D}$ at a stage of pregnancy when endothelial proliferation has disappeared. Nonkey C 679 , one hundred and second day of pregnancy. $\times 25$. 

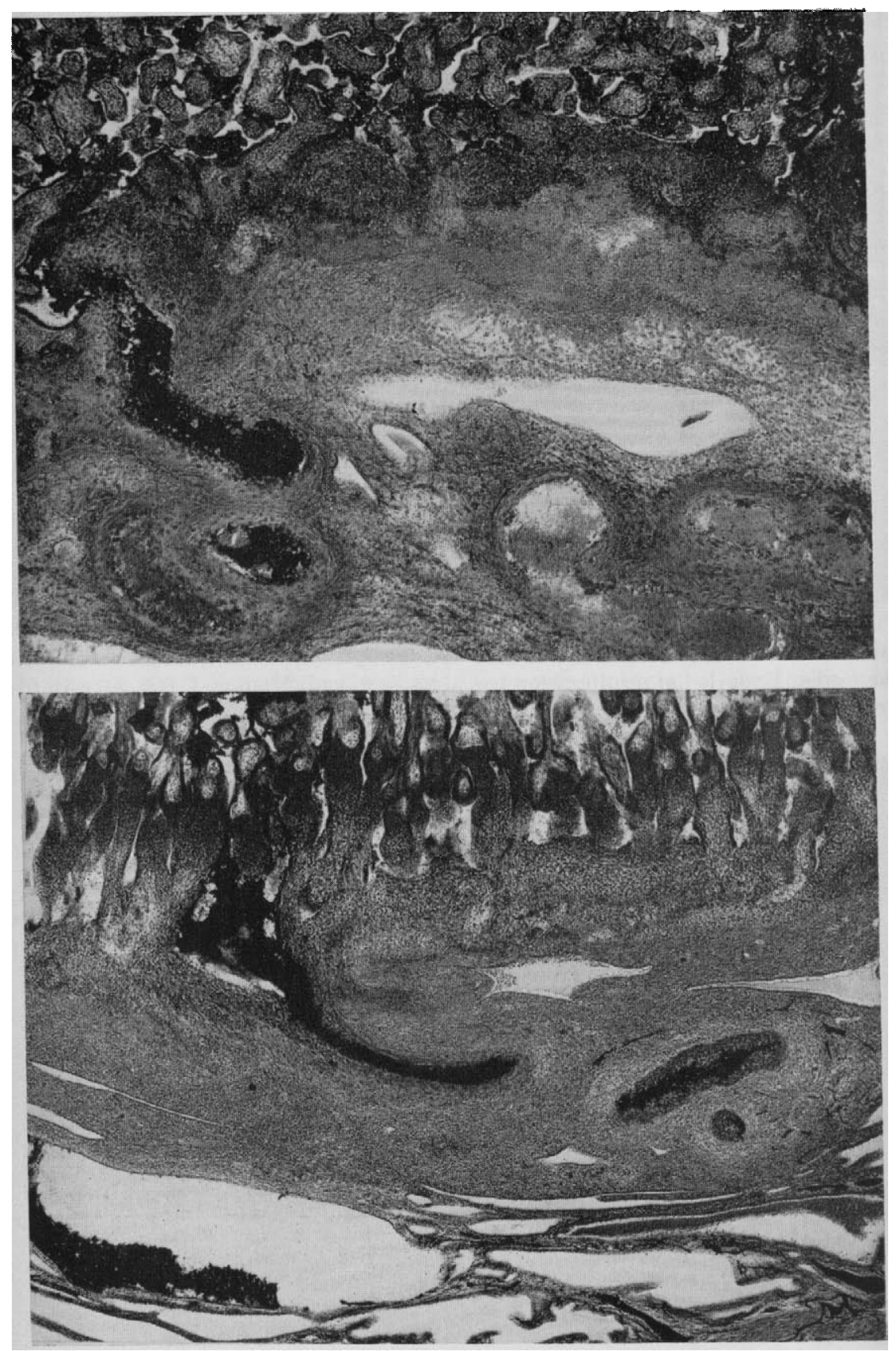

Fig. 11. Below, the arterial entry seen in fig. 8 is reproduced for comparison with a similar entry (shown above) in a human placenta from the twenty-second week of pregnancy. Monkey C 658. $\times 30$. Human specimen 8593. $\times 30$. 
alteration in the arteries requiring notice is an intimal fibrosis which makes its appearance about the time of conversion and persists to term. Like the other changes it is first seen at the placental junction and progresses proximally. At term it is pronounced in the myometrial arcuate arteries. In degree the process varies irom an insignificant narrowing of the lumen to actual occlusion of many small branches and an occasional main stem (fig. 10).

The details and the chronology of the transformations just described are based upon observations in the monkey. A similar time table and descriptions are currently being worked out in the human. So far only a qualitative statement is possible to the effect that the histological alterations and proximal progression of changes follow similar lines in the two species of primates. Figure 11 illustrates this similarity by presenting a section from a monkey placenta and one from a human side by side.

\section{Discussion}

Drawing all the foregoing factors together into a continuous story from which certain deductions regarding physiology may be made, it is seen that during the early, limited period of uterine growth the arterial bed increases rapidly in size and length and in addition produces large quantities of extra building units for future utilization. Subsequently, in response as Reynolds has shown to the growth stimulus of ever increasing uterine tension (14), these units hypertrophy and undergo orderly arrangement, further increasing arterial length and size. Even this, however, as was pointed out some years ago when the phenomena under consideration were first described $(10)$, is still a preparatory period for the arteries, for their great length, now stored in coils and loops throughout the endometrium, is being held in reserve for the final era when, its growth substantially at an end, the uterus commences stretching and the arterial coils are paid out.

Meanwhile certain useful effects are achieved as by-products during the intermediate stages. Thus, the early piling up of hyperplastic endothelium in the arterial lumen, with resultant diminution of lumen diameter, acts as a control device, reducing the flow of blood into the intervillous space, while maintaining pressure. Similarly, the coiling of the arteries in the early months provides a slowing mechanism which, however, keeps the pressure at a level closer to the original maternal blood pressure than would be the case were the arterial channels labyrinthine or richly arborized.

The intimal fibrosis, insofar as it does not overstep the bounds of the physiological and limit afferent blood flow to a pathological extent, is another control mechanism which regulates the volume of blood entering the placenta. It is also a phenomenon which has long been recognized as a safeguard against serious hemorrhage at parturition.

\section{CIRCULATION IN THE INTERVILLOUS SPACE}

It was stated, in comparing the intervillous space to a capillary, that the trophoblast covering the chorionic villi forms the wall of the space in the same way that the endothelium forms the wall of a capillary. Some explanation of this 


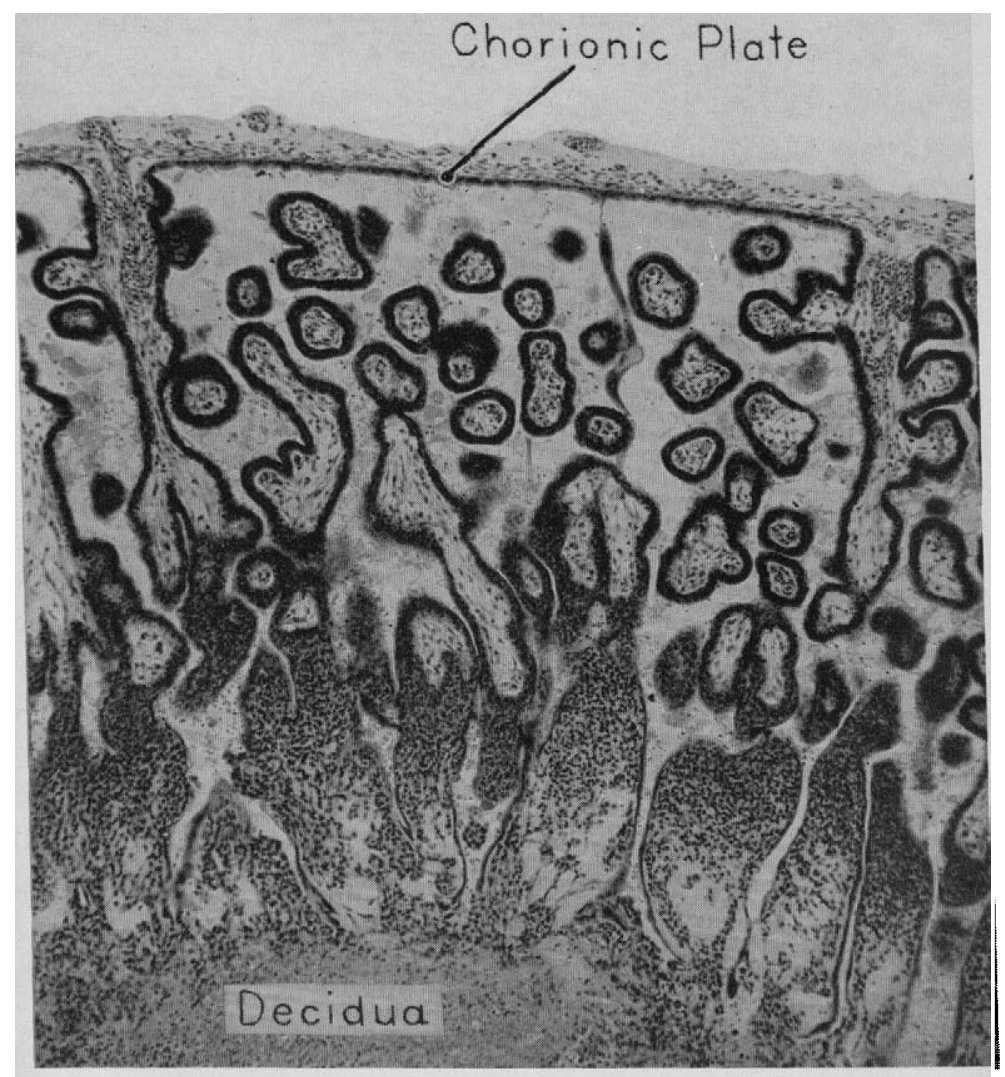

FIg. 12. A portion of the uterine wall and attached placenta in the monkey to show the manner in which the trophoblast lines the intervillous space and invests the chorionic villi. The latter have their "roots" in the chorionic plate and hang down into the intervillous space like an inverted tree. $\mathrm{C} 477$. Twenty-ninth day of pregnancy. $\times 50$.

arrangement in the hemochorial placenta may be in order for the benefit of those more familiar with the ungulate or rodent placenta than the primate.

The intervillous space with which the endometrial vessels connect, in the manner we have seen, develops out of intercommunicating vacuoles in the outer syncytial layer of the wall of the implanting blastocyst (8). The space, therefore, is lined in the first instance by syncytio-trophoblast (fig. 12). Very early, solid columns of tissue arising from the inner portion of the blastocyst wall grow into the space pushing the syncytium ahead of them like a covering. These columns have a core of fetal mesoderm and are bordered by cytotrophoblast (Langhans cells). Branches of the fetal blood vessels grow into the mesodermal cores. The whole unit, composed of core and border and fetal vessels, is the chorionic villus (fig. 13) and, since rilli branch luxuriantly as they grow, many are always caught in cross section in microscopic preparations of the placenta and it is easy to forget that the surface of each one is part of the wall of the intervillous space as a whole. In early formative stages, as described, therefore, two layers of trophoblast, the syncytium and the cytotrophoblast, intervene between the maternal blood in 


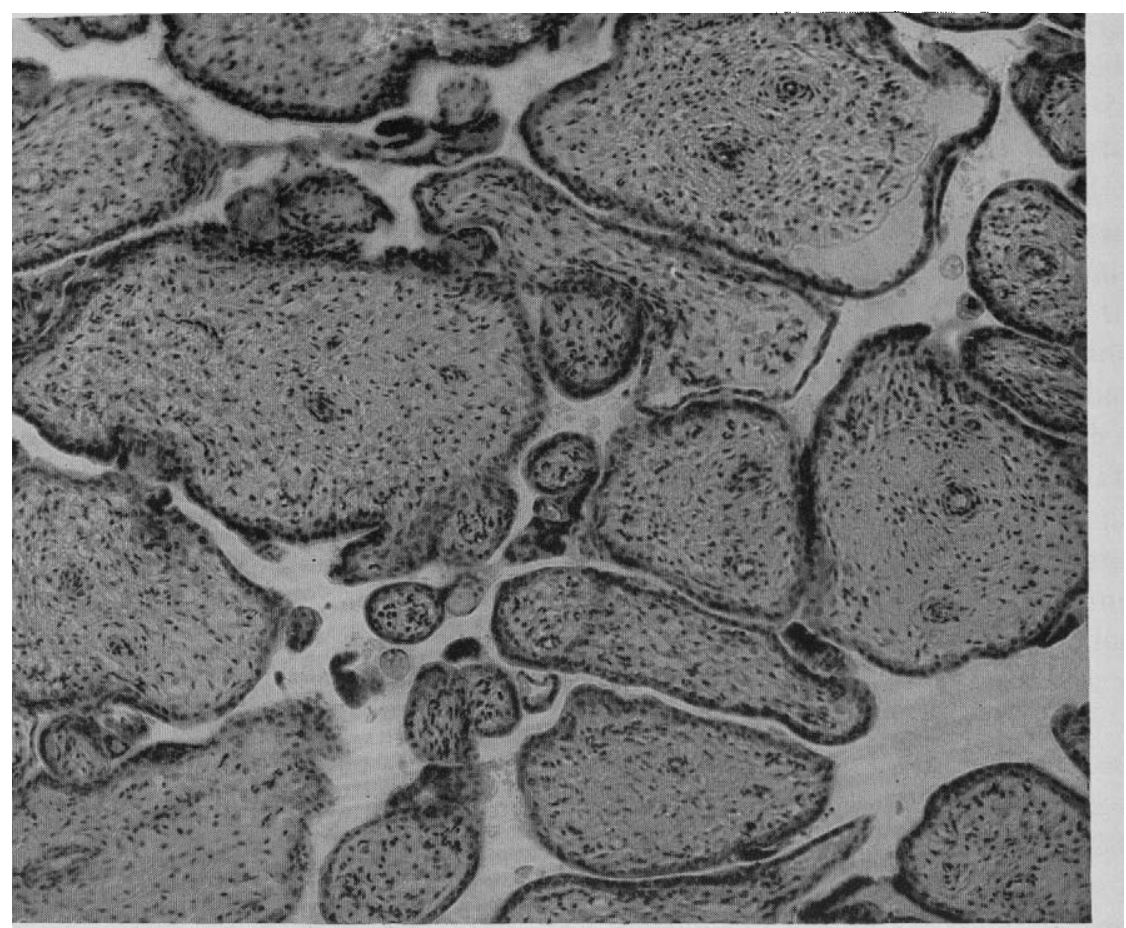

FIG. 13. A photomicrograph of cross sections of several human chorionic villi in the twelfth week of pregnancy. Fetal vessels are seen in the mesodermal core of the villi. Langhans cells and attenuated syncytium clothe the villi. $7597 . \times 100$.

the intervillous space and the mesoderm surrounding the fetal capillaries. Later the cytotrophoblast disappears and the syncytium becomes very much attenuated so that the barrier to diffusion is greatly reduced.

Maternal blood is kept circulating within the intervillous space essentially by the high pressure in the maternal arteries. This, exceeding the pressure in the intervillous space by 60 to $70 \mathrm{~mm} \mathrm{Hg}(15,16)$, causes arterial blood to enter in actual spurts which carry the blood high up toward the fetal surface before lateral seepage occurs. Thereafter the blood converges upon the orifices leading into the maternal veins. The controlling hemodynamic force which effects the circulation in the intervillous space is aided by a number of anatomical and physiological arrangements such as the placental septa, myometrial contractions, the villous pulse, etc. These were previously considered the decisive factors in maintenance of the circulation (17-19), but recent observations indicate that this role is played by the hemodynamic force.

\section{FETAL PLACENTAL CIRCULATION}

In the mature placenta the umbilical cord with its contained arteries and vein enters the placenta through the chorionic plate which forms the fetal surface of that organ. The chorionic villi have their roots, so to speak, in this plate and hang downward into the intervillous space like an inverted tree. This concept has long 


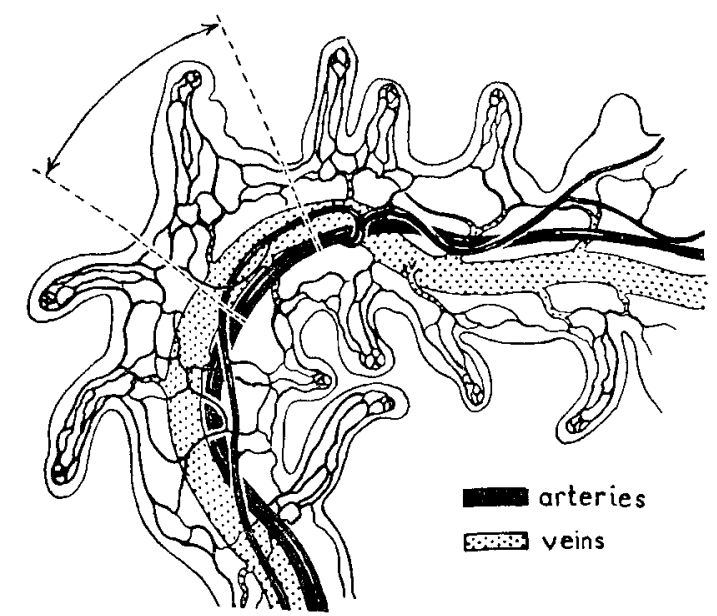

\section{Superficial capillary network}

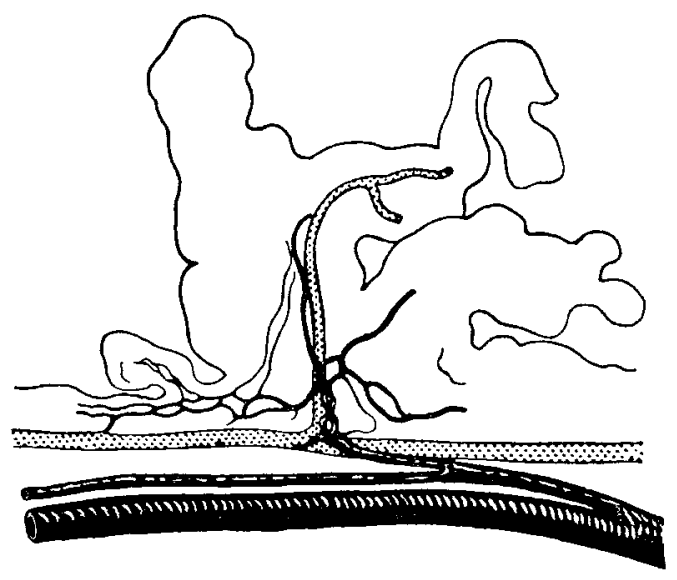

\section{Paravascular network}

Fig. 14. Schematic representations of the course of fetal blood vessels in the chorionic villi of the human placenta, after $\mathrm{B} \varnothing \mathrm{e}(20)$. In the upper drawing the vasculature is shown as it would appear if the paravascular network had been dissected away. The points at which the network would attach to the stem vessels are indicated by the broken lines.

been held and was recently confirmed by Bøe (20), Romney (21), and others and by Reynolds (22) on the physiological side. In Finn Bøe's injection study it is shown, as illustrated in figure 14, that main trunks of fetal arteries rumning in the chorionic and subchorionic plate give rise to a few long branches of wide diameter which travel down the villous stems. Sub-branches of these are of two orders: those which feed a superficial capillary network within terminal villi and those which feed a paravascular network providing extra-villous shunts from artery to vein which act as safety valves to prevent overloading of the villous circu- 
lation. Collecting venules and veins, relatively less numerous than arteries, transmit the aerated blood back to the fetus via the umbilical rein. Reynolds' studies (22) upon the fetal sheep show how the circulation is maintained in this system. Essentially, just as the circulation in the maternal placenta is dominated by the vis-a-tergo of maternal blood pressure, so the fetal placental circulation is maintained by the head of fetal blood pressure. Arterial blood is transmitted to the umbilical artery in particularly favorable volume and under high pressure by virtue of the special anatomical arrangements of the circulation within the fetus itself. The pressure continues high until about the level of the paravascular plexus since it travels down the villous stems through rather wide channels where little energy is lost through friction. It is also high in the umbilical vein, being so maintained by the action of a sphincter at the start of the ductus venosus. The pressure fall betwcen umbilical artery and vein is not more than 35 or $40 \mathrm{~mm}$ $\mathrm{Hg}$. To quote Reynolds, "the placental capillary vessels must be under higher pressure than other capillary vessels throughout the body" and the balance maintained between their pressure and that in the intervillous space, which does not exceed $10 \mathrm{~mm} \mathrm{Hg}$ between contractions, is highly favorable to transfer of water from fetus to mother.

Transfer in the opposite direction, i.e., from mother to fetus, has been investigated by many workers in numerous ways and with respect to a multitude of substances. With particular clarity the work of Flexner and his associates (23), using tracer substances, has revealed the decisive role played by the degree of efficiency of the maternal circulation in the placenta at any given stage of pregnancy, recalling once more the initial thesis of this report.

This brief resume of the interactions of the maternal and fetal circulations at the capillary level demonstrates that the elaborate vascular provisions on both sides are all geared to serve the requirements of this ultimate functioning area. The manner in which the final arterial pattern in the mature placenta develops shows not only that the pattern of the vessels is intimately related to the function of the organ but that the vessels are remarkably adaptable to changes in functional requirements. This is perhaps the most outstanding over-all attribute of placental vasculature, the great complexity of the adaptations of the endometrial arteries being particularly remarkable in that they are characteristically made in anticipation of distant future needs.

\section{SLMMARY}

1. The placenta has been presented as an organ well adapted for study of those basic functions of metabolic exchange usually associated with systemic capillary beds alone.

2. The analogy between the intervillous space of the primate placenta and a systemic capillary has been demonstrated from the morphological and physiological standpoints.

3. The endometrial blood vessels have been analyzed to show their protean adaptability to changing functional demands during the menstrual cycle and pregnancy.

4. The circulatory mechanies of both the maternal and the fetal placental vas- 
culature and their interaction at the level of the intervillous space have been described.

\author{
Dept. of Embryology \\ Carnegie Institution of Washington \\ Baltimore, $M d$.
}

\title{
BIBLIOGRAPHY
}

1. Daron, G. H.: The arterial pattern of the tunica mucosa of the uterus in Macacus rhesus. Am. J. Anat., 58: 349, 1936.

2. Darox, G. H.: The veins of the endometrium (Macacus rhesus) as a source of the menstrual blood. Anat. Rec., 67: suppl. 3, 13, 1937.

3. MARKeE, J. E.: Menstruation in intraocular endometrial transplants in the rhesus monkey. Carnegie Inst. Wash. Pub. 518, Contrib. to Embryol,, 28: 219, 1940.

4. Bartelimez, G. W.: The human uterine mucous membrane during menstruation. Am. J. Obst. \& Gynec., 21: 623, 1931.

5. Bartelmez, G. W.: The mechanism of menstruation. Anat. Rec., 97: 380, 1947.

6. Bartelmez, G. W.: Cyclic changes in the endometrium of the rhesus monkey (Macaca mulatta). Carnegie Inst. Wash. Pub. 592, Contrib. to Embryol., 34: 99, 1951.

7. Bartelmez, G. W.: Personal communication.

8. Wislocki, G. B., ANd Streeter, G. L.: On the placentation of the macaque (Macaca mulatta), from the time of implantation until the formation of the definitive placenta. Carnegie Inst. Wash. Pub. 496, Contrib. to Embryol., 27 : 1, 1938.

9. RAMSEx, E. M.: The vascular pattern of the endometrium of the pregnant rhesus monkey (Macaca mulatta). Carnegie Inst. Wash. Pub. 583, Contrib. to Embryol., 33: 113, 1949.

10. Ramsey, E. M.: Venous drainage of the placenta of the rhesus monkey (Macaca mulatta). Carnegie Inst. Wash. Pub. 603, Contrib. to Embryol., 35: 151, 1954.

11. Ramsey, E. M.: Circulation in the maternal placenta of primates. Am. J. Obst. \& Gynec., 67: 1, 1954.

12. Reynolds, S. R. M.: Uterine accommodation of the products of conception: physiologic considerations. Am. J. Obst. \& Gynec., 53: 901, 1947.

13. OKKELS, H., AND ENGLE, E. T.: Studies on the finer structure of the uterine blood vessels of the Macacus monkey. Acta pathol. et microbiol. scandinav., 15: 150, 1938.

14. ReYnolds, S. R. M.: The relation of hydrostatic conditions in the uterus to the size and shape of the conceptus during pregnancy: a concept of uterine accommodation. Anat. Rec., 95: 283, 1946.

15. Woodbury, R. A., Hamilton, W. F., and Torpin, R.: The relationship between abdominal, uterine and arterial pressures during labor. Am. J. Physiol., 121: 640, 1938.

16. Alvarez, H., and Caldeyro, R.: Contractility of human uterus recorded by new methods. Surg., Gynec. \& Obst., 91: 1, 1950.

17. Stieve, H.: Bemerkungen ueber den Blutkreislauf in der Placenta des Menschen. Zntrlbl. f. Gynaekol., 65: 370, 1941.

18. Spanner, R.: Muetterlicher und kindlicher Kreislauf der menschlichen Placenta und seine Strombahnen. Ztschr. f. Anat. u. Entwickl., 105: 163, 1935.

19. Bumm, E.: Ueber die Entwicklung des muetterlichen Blutkreislaufes in der menschlichen Placenta. Arch. f. Gynaekol., 43: 181, 1893.

20. BøE, F.: Studies on the vascularization of the human placenta. Acta obst. et gynec. scandinav., 32: suppl. 5, 1, 1953.

21. Romxey, S. L., AND Reid, D. E.: Observations on the fetal aspects of placental eirculation. Am. J. Obst. \& Gynec., 61: 83, 1951.

22. Reynolds, S. R. M.: Hemodynamic characteristies of the fetal circulation. Am. J. Obst. \& Gynec., 68: 69, 1954.

23. Flexner, L. B., AND Gellhors, A.: The comparative physiology of placental transfer. Am. J. Obst. \& Gynec., 43: 965, 1912. 


\section{DISCUSSION}

\section{GARMAN H. DARON, Ph.D.}

I see that instead of one, Dr. Ramsey has given us three vascular patterns to contemplate upon; the fetal circulation within the placental villi, the circulation involving the intervillous space and the vascular pattern in the endometrium of the non-pregnant state. It is always pleasing to hear, to read and to study Dr. Ramsey's papers because of the clarity of description of her observations and her carefully considered deductions.

The vascular pattern within the endometrium exhibits some unique features. However, perhaps there is some background of fundamental physiological vascular anatomy here, common with other vascular patterns in the animal organism. The nature of this Conference provides an excellent opportunity to point out some problems suggested by Dr. Ramsey's paper in the hope that through discussion some additional light may be thrown upon them.

First, I would like to call your attention to the coiled, spiral arteries within the endometrium. Dr. Ramsey has well demonstrated that the presence of this great arterial length is utilized in the pregnant uterus during the period of conversion. While this is true may not this same anatomical feature serve more than one function either simultaneously or in sequence in time? Is there objective evidence that the greatly coiled nature of these arteries, so well seen in the illustrations just presented or perhaps in somewhat similar patterns elsewhere in the organism, actually have a function in reducing blood flow or blood pressure at their terminal ends either in the early placenta before conversion, or in the late stages in the menstrual cycle? For those of you not intimately familiar with these endometrial vessels let me state that they are almost as highly coiled in the late stages of the menstrual cycle and that the vessels in question have a luminal diameter of about 30 to 45 micra in the macaque non-pregnant uterus.

Again because of the interests of this assembled group and your familiarity with fluid loss this is an excellent opportunity to raise the question as to how is the intra- and intercellular fluid removed in the regression stage of the menstrual cycle on which so much rests in the theory of the mechanism of menstruation that Dr. Ramsey outlined? Dr. Markee has frequently pointed out this problem but as yet there is no completely satisfying answer. This is complicated by the observations of Drs. Wislocki and Dempsey that there are no, or at least a very limited supply of lymphatics in the superficial portion of the endometrium. Vitally involved in this connection is the problem of the existence of arteriovenous anastomoses in this superficial zone of the endometrium. While personally, in study of serial sections of this region of the macaque uterus I have never seen direct arteriolar feeding of the dilated venous lakes, I would like for Dr. Ramsey to elaborate on this point of endometrial arterio-venous anastomoses.

$$
\begin{aligned}
& \text { Dept. of Anatomy } \\
& \text { University of Oklahoma }
\end{aligned}
$$

